\title{
Investigation on the mechanism of enhancing heat transfer in a pebble bed by adding in the non-fixed number of smaller-sized spheres
}

\author{
Leisheng Chen ${ }^{1 *}$, Jiahao Zhao ${ }^{1}$, Yuejin Yuan ${ }^{1}$, and Jaeyoung Lee ${ }^{2}$ \\ ${ }^{1}$ College of Mechanical \& Electrical Engineering, Shaanxi University of Science \& Technology, Xi' an, 710021, China \\ ${ }^{2}$ School of Mechanical \& Control Engineering, Handong Global University, Pohang, 37554, South Korea
}

\begin{abstract}
Enhancing heat transfer in the pebble bed reactor could reduce the surface temperatures and lower the possibility of forming hot-spots. The effectiveness of inserting a smaller sphere into a structured pebble bed on optimizing the heat transfer has been confirmed, and yet, the mechanism of heat transfer enhancement is still not fully understood. The impact of the quantity and size of the small spheres on the heat transfer characteristics has been investigated in this study and the mechanism of enhancement was analyzed. It was found that: (1) When the volume or the surface area of the inserted sphere was kept the same, the overall heat transfer coefficients (HTC) of the pebble bed in case 2 or case 3 respectively demonstrated $1.4 \%$ or $2.8 \%$ higher than that of the bed in case 1 ; $(2)$ the overall HTC showed an increasing trend with the decreasing ratio of the surface area to the volume; (3) the varying trends of local HTCs along the designated direction were similar among 3 cases and the strongest heat transfer positions were found near pebble-sphere contact points. Such findings will help to design a better pebble bed core.
\end{abstract}

\footnotetext{
* Corresponding author:1sheng.ch@sust.edu.cn
} 


\section{Introduction}

The pebble bed in a Pebble-bed type High Temperature Gas-cooled Reactor (HTGR) is composed of a large number of randomly stacked spherical fuel elements, and the contact between any two fuel elements may cause hot-spots appearing on the pebbles' surfaces [1]. Once the temperature of hot spots gets too high, the structural integrity of the fuel elements may get affected, which may result in a break-apart of the spheres and lead to a severe accident like a release of fission products [2]. To reduce the possibility of hotspots appearance, researchers have studied the heat transfer characteristics of different pebble beds [3-5]. Moreover, Forsberg and Peterson [6] proposed that packing a bed with multi-sized pebbles rather than mono-sized pebbles could help the reactor reach a higher power density and also enhance the heat transfer of the bed. However, thus far, the research on the heat transfer characteristics of beds packed with multi-sized spheres has barely been conducted. In our previous study, Chen and Lee [4,7] have analyzed the impact of the diameters of the pebble and the inserted sphere on the heat transfer characteristics of a face-centeredcubic (FCC) structured pebble bed and concluded that compared to the pebble bed without a small sphere, placing a sphere in the bed could reduce the surface temperature of certain pebbles and therefore increase the average heat transfer coefficient (HTC); besides, it was found that the larger the inserted sphere was, the stronger the enhancement would be. Although the reason for this heat transfer enhancement was partly described as the expansion of effective heat convection area caused by the inserted sphere contacting the adjacent pebbles, still, more and deeper mechanisms need to be discovered in order to apply this approach to a real pebble bed reactor. Therefore, in the present study, the impact of the number of inserted spheres, volume, and surface area on the heat transfer characteristics is investigated in details. The size and quantity of inserted spheres are determined by controlling the volume or surface area constant, and 3 cases (two spheres with a diameter of $0.03 \mathrm{~m}$, one sphere of $0.0378 \mathrm{~m}$, and one sphere of $0.0424 \mathrm{~m}$ ) are compared to find out which parameter is more critical. This study provides useful data and will make contributions to a better design of an HTGR core.

\section{Analysis and modeling}

In this study, the numerical method is applied and the previously created CFX model [8] is used. The mechanism of how inserted sphere(s) affected the heat transfer characteristics of the pebble beds and what was the dominant parameter were investigated in details using this validated numerical model. A total of 3 cases were designed for this purpose, for the first case, two spheres with a diameter of $0.03 \mathrm{~m}$ were symmetrically placed in the void of the FCC structured pebble bed; to keep the volume of the solid or heat-transfer surface area the same, one sphere with a diameter of $0.0378 \mathrm{~m}$ or $0.0424 \mathrm{~m}$ was respectively inserted into the bed for the second and third case. The small spheres were made of the material as the pebble but without internal heat generation.

\subsection{Geometry}

As shown in Fig. 1a, the geometry consists of a fluid part (air) and a solid part (brass). The duct represents the fluid, and the solid part is composed of 14 pebbles, 14 heaters, and a few inserted balls. Relevant parameters of the components are listed in Table 1.
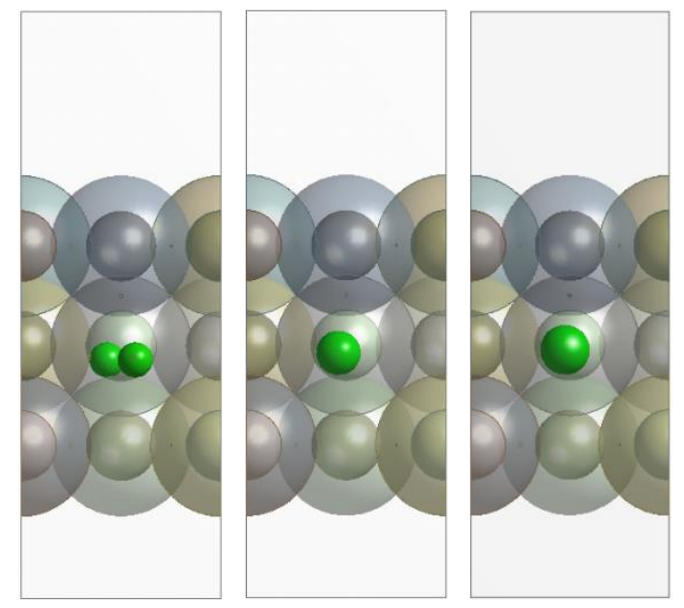

(a)

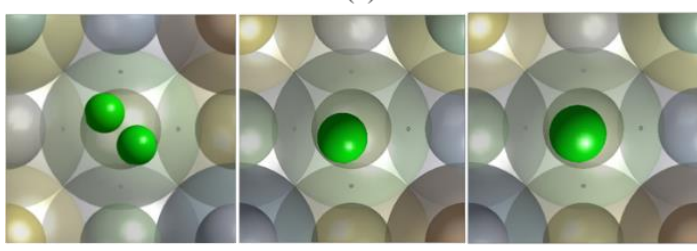

(b)

Fig. 1. (a) Geometry of FCC pebble-bed (b) Position demonstration of the small spheres

The side and top views of the positions of small spheres in the pebble bed are clearly described in Fig. $1 \mathrm{a}$ and $\mathrm{b}$. For the first case, two small spheres were diagonally and symmetrically placed; for the second and third cases, the sphere was inserted in the left side of the void. The spheres in cases 1 and 2 had the same volume, while had the same surface area for cases 1 and 3. Every small sphere contacted 3 surrounding pebbles and the diameter of each contact point was limited to $2 \mathrm{~mm}$.

Table 1.Parameters of the components

\begin{tabular}{|c|c|c|c|c|c|}
\hline \multicolumn{3}{|c|}{ Solid } & \multicolumn{3}{c|}{ Fluid } \\
\hline $\begin{array}{c}\text { Pebble[ } \\
\mathrm{m}]\end{array}$ & $\begin{array}{c}\text { Heater[ } \\
\mathrm{m}]\end{array}$ & $\begin{array}{c}\text { Sphere } \\
{[\mathrm{m}]}\end{array}$ & $\begin{array}{c}\text { Width[ } \\
\mathrm{m}]\end{array}$ & $\begin{array}{c}\text { Depth[ } \\
\mathrm{m}]\end{array}$ & $\begin{array}{c}\text { Height[ } \\
\mathrm{m}]\end{array}$ \\
\hline 0.12 & 0.06 & $\begin{array}{c}0.03- \\
0.0424\end{array}$ & 0.17 & 0.17 & 0.5 \\
\hline 14 & 14 & $1-2$ & - & - & - \\
\hline
\end{tabular}




\subsection{Meshing}

The constructed high-quality mesh is shown in Fig. 2ad. The mesh size $\left(2 \times 10^{-3} \mathrm{~m}\right)$ and quality for the fluid, the pebbles, and the heaters are the same in 3 cases, the only difference comes from the mesh quantity of the inserted spheres. By applying 5 boundary layers on both sides of the fluid-pebble interfaces, the thermal field can be analyzed more accurately. The total numbers of nodes and elements are around 3 million and 14.79 million, respectively, and the meshindependence test was already done elsewhere [4].

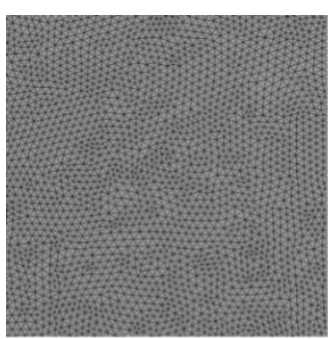

(a)

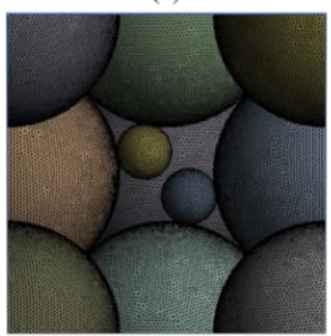

(c)

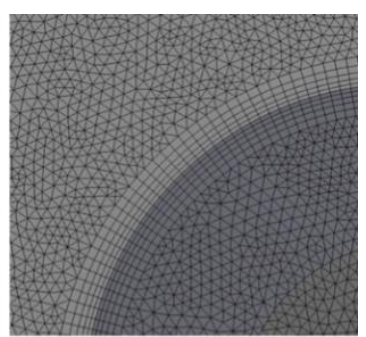

(b)

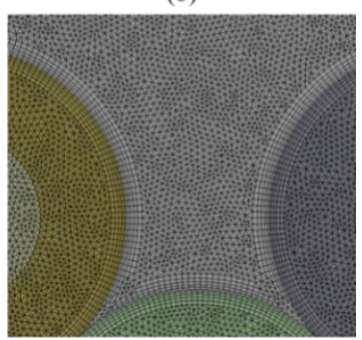

(d)
Fig. 2. Meshes of (a) the fluid (b) the fluid-solid interface (c) the small spheres and (d) the pebbles

\subsection{Boundary conditions}

The boundary conditions in these numerical simulations were determined directly from the physical properties and operational parameters of the High Temperature Reactor-Pebble bed Modules (HTR-PM) based on the similarity principle. It is worth mentioning that the pebble diameter was scaled up by 2 times $(12 \mathrm{~cm})$ to better observe the flow patterns of the coolant in the pebble bed in the experiments. The $\mathrm{k}-\varepsilon$ turbulence model and a turbulence intensity of 5\% were selected; the inlet velocity of the coolant was set to $2.1 \mathrm{~m} / \mathrm{s}$ by calculation, and the coolant enters the test area with a temperature of $298.15 \mathrm{~K}$; the power generation by each heater was $1.0 \times 10^{6} \mathrm{~W} / \mathrm{m}^{3}$.

\subsection{Measurement locations}

The heat transfer characteristics of two designated planes, the middle plane (colored in grey) and the diagonal plane (colored in red) which cut through the inserted small spheres as shown in Fig. $3 a$ and $b$ were specifically investigated. For this purpose, at these two planes, the measurement positions on the surfaces of the spheres were designed. At the middle plane, because placing only one sphere $(D=0.0378 \mathrm{~m}$ or $0.0424 \mathrm{~m}$ ) made the pebble bed structure no longer symmetrical, a total of 46 measurement positions distributed on both the left and the right side of the void, as shown in Fig. 3c, were used to ensure the accuracy of the numerical results. Among these locations, No. 1 to 6,7 to 17 , and 18 to 23 were located at the top, middle, and bottom pebble, respectively. At the diagonal plane, measurement positions No. 1 to 11 and No.12 to 22 were located on the top and bottom pebble beds, respectively, as shown in Fig. $3 \mathrm{~d}$. It is worth mentioning that the orientations of lines No. 1, 11,12 , and 22 in the left half were shifted by $2^{\circ}$ from the vertical or horizontal direction to avoid pebblepebble and pebble-sphere contact points where the heat transfer was determined not by convection but by conduction. However, lines No. 7 and 30 were not taken into consideration during the data analysis because they were located too close to the pebblepebble contact area.

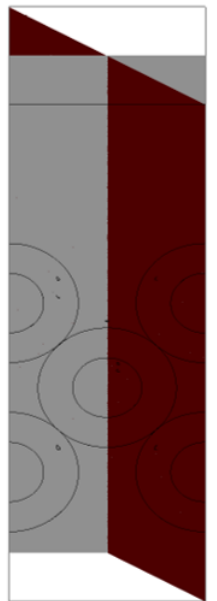

(a)

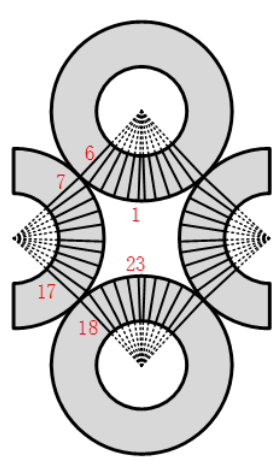

(c)

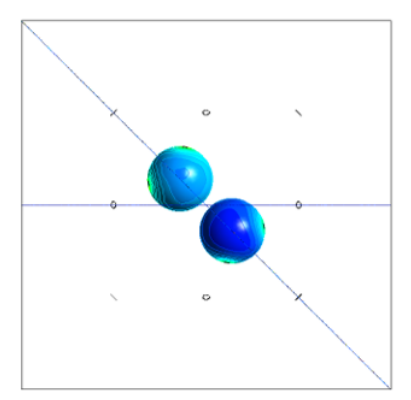

(b)

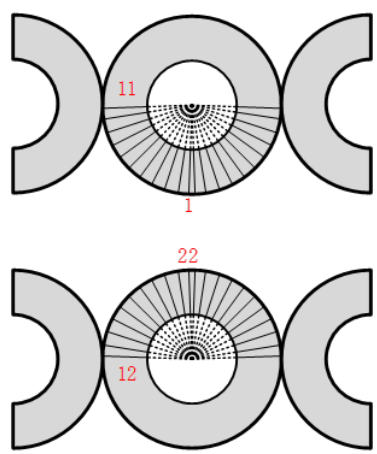

(d)
Fig. 3. (a) The middle plane (grey)and the diagonal plane (red); (b) the top view of relative positions of two designated planes and small spheres; (c) measurement locations on the middle plane; (d) measurement locations on the diagonal plane

The temperature of all specified positions and $5 \mathrm{~mm}$ below the surface were measured, and the average HTC is calculated using the following formula:

$h_{\text {avg }}=\sum_{i=1}^{88}\left(\frac{k_{\text {solid }} \Delta T_{i}}{r^{2}\left(\frac{1}{r_{2}}-\frac{1}{r_{1}}\right)} \times \frac{1}{T_{\text {surf }}-T_{\infty}} \times \frac{1}{88}\right)\left(W / m^{2} K\right)$

Where $\mathrm{k}$ is thermal conductivity; $\Delta \mathrm{T}_{\mathrm{i}}$, the temperature difference between pebble surface and 5 $\mathrm{mm}$ beneath at the $i_{\text {th }}$ measurement point, $T_{\text {surf }}$, the surface temperature of the pebble; and $\mathrm{T}_{\infty}$, the fluid 
temperature at the inlet; $r, r_{1}$, and $r_{2}$ are the radius of the pebble, distance from pebble center to the surface, and distance from pebble center to $5 \mathrm{~mm}$ beneath the surface, respectively.

\section{Results and discussion}

The mechanism of heat transfer enhancement and the impact of the number of spheres on the heat transfer characteristics of a pebble bed were investigated through numerical simulation. By controlling the number, the volume, and the surface area of the spheres, the local and average heat transfer coefficients of the pebble bed in the middle plane and diagonal plane were studied in detail under 3 conditions $(D=0.03 \mathrm{~m}$ of two spheres for case $1, D=0.0378 \mathrm{~m}$ of one sphere for case 2 , and $\mathrm{D}=0.0424 \mathrm{~m}$ of one sphere for case 3 ).

\subsection{Average HTCs of the pebble beds}

Fig. 4a shows the average HTCs of both the middle (black open circles) and the diagonal planes (red open circles) of all 3 cases. The black solid circles represent the average heat transfer coefficients by averaging those obtained from both the middle and diagonal planes. It can be seen that 1) the HTC values at the diagonal planes were smaller than those at the middle planes for all 3 cases; 2) case 2 demonstrated a $1.4 \%$ higher average heat transfer coefficient than case 1 even though two cases had the same sphere volume; 3 ) similarly, case 3 showed an average HTC increase of $2.8 \%$ compared to case 1 when the surface area was kept the same. Heat transfer enhancement can be found as the number or diameter of the sphere changes, however, the enhancement is quite small. The possible reasons for the above findings will be discussed in details in the following sections.

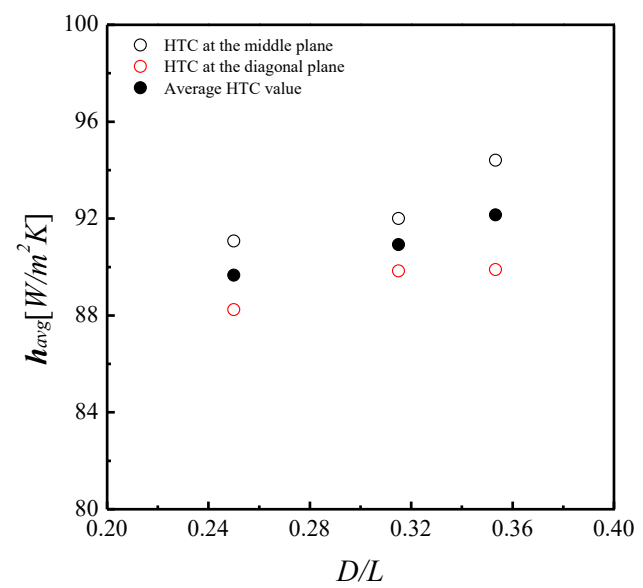

(a)

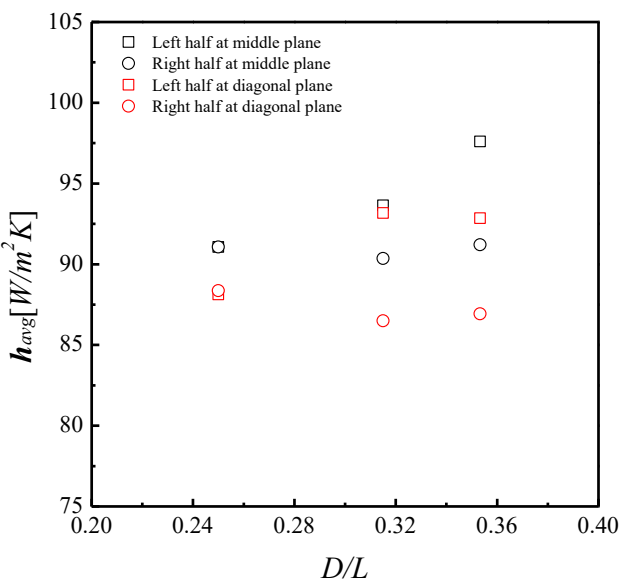

(b)

Fig. 4. (a) Average HTCs of all 3 cases (b) Average HTC values at different planes

As the ratio of the surface area to the volume decreased from case 1 to case 3 , however, it was found that the heat transfer coefficient did not show a similar decreasing trend as presented by Forsberg and Peterson [6]. The reason might be that the small spheres were not generating heat and only worked as the expanded surfaces to adjacent pebbles, which were able to enlarge the convective surface area and therefore result in a better convective heat transfer.

Heat transfer characteristics at both the left half and the right half of the middle and diagonal planes were analyzed and the obtained HTCs plotted in Fig. 4b. It can be seen that for cases 2 and 3 that contained only one small sphere, the HTC values at the left and right half of the plane were not the same, and the half containing the small sphere had a higher average HTC. However, such discrepancy was not found in the pebble bed with two same-sized spheres because of the symmetric structure. It is worth discussing more on this point to understand the mechanism more clearly for possible application to a pebble bed core design.

\subsection{Local HTCs in the pebble beds}

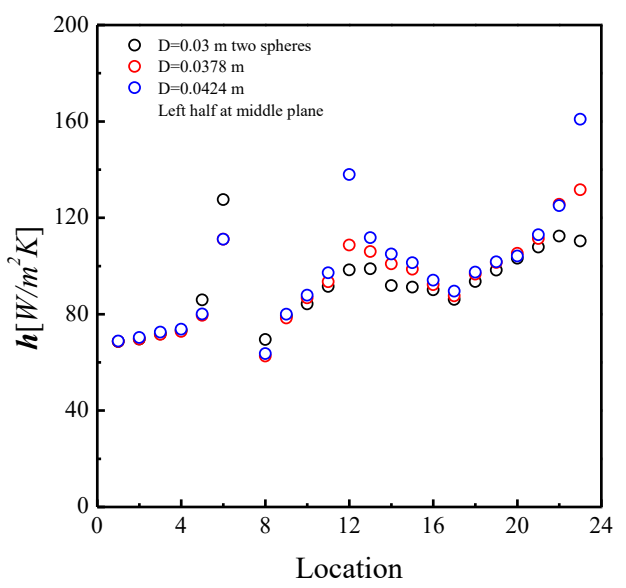

(a) 


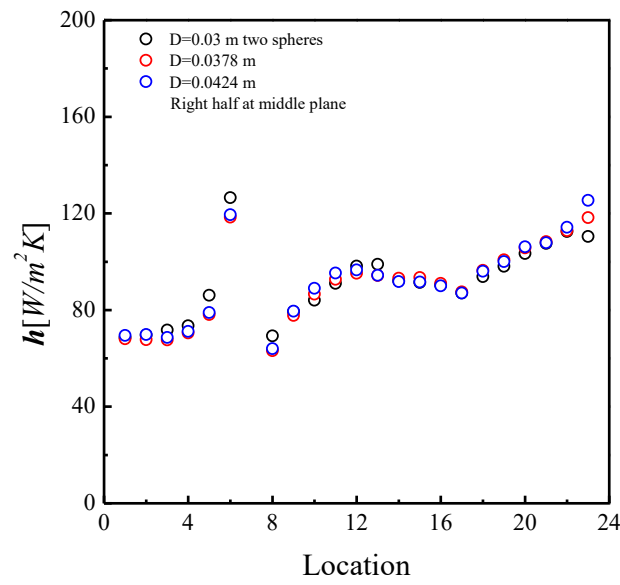

(b)

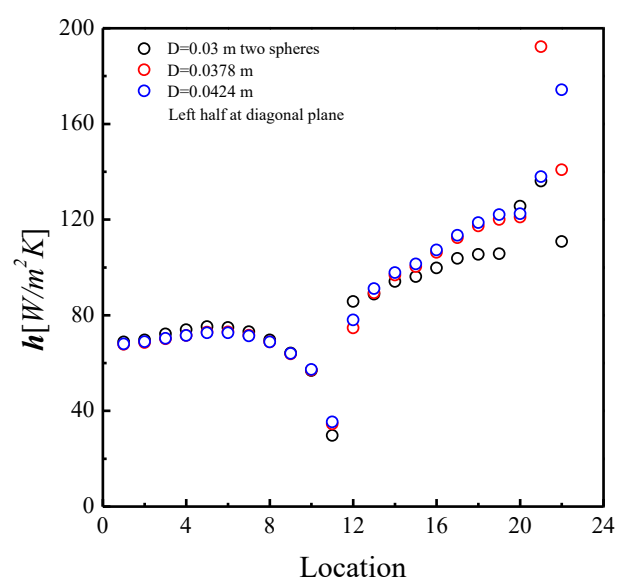

(c)

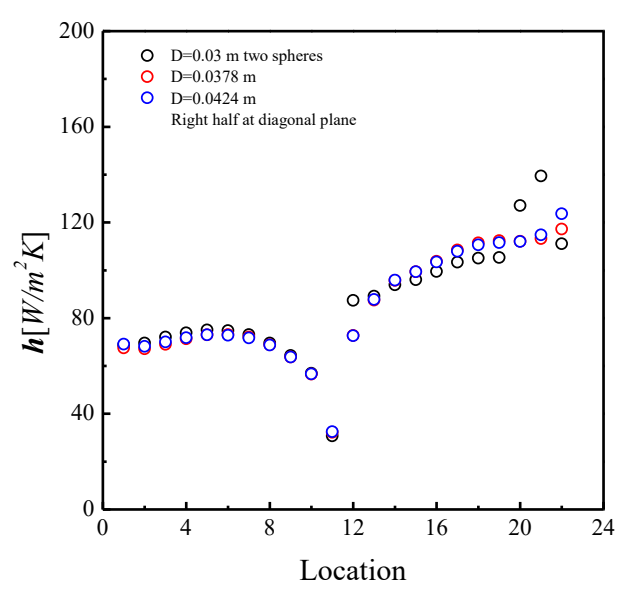

(d)

Fig. 5. Local HTCs of all 3 cases at (a) the left half (b) the right half of the middle plane (c) the left half and (d) the right half of the diagonal plane.

The local HTCs at the middle planes of all 3 designated cases were compared to find the reason for heat transfer enhancement and the results were plotted in Fig. 5a, b, c, and d. The varying trends at the middle plans were found similar among 3 cases as shown in Fig. 5a. In terms of local heat transfer intensity, particularly from lines No. 10 to 23 , case 3 showed the best heat transfer intensity, and second to it was case 2 . It is believed that the enhancement was caused by the sphere parameter as well as sphere-pebble contacts. The heat generated in the pebbles was out-dissipated via the spheres, and the larger the sphere size is, the more the transferred heat will be. As shown in Fig. 5b, the heat transfer in cases 2 and 3 is almost the same because the influence of the small sphere on the right half of the plane is limited. The impact of the inserted small sphere became stronger as the measurement point got nearer to the bottom pebble, therefore, case 3 showed the best heat transfer characteristics and case 2 came second especially at location No. 23 as the same as the characteristics at the left half of plane. Although the contact points were differently located because the diameters of the spheres differed from each other, the fact that contact enhancing heat transfer did not change.

The local HTCs of the left and right half of the diagonal plane are plotted in Fig. $5 \mathrm{c}$ and 5d. As shown in Fig. 5c, the heat transfer coefficient increases slightly from location No. 1 to 7 , and then it starts to decrease until the pebble-pebble contact point (Location No. 11). As for the bottom pebble, the weakest heat transfer takes place near the pebblepebble contact point (Location No. 12), and the local HTCs are getting larger and larger when the measurement point goes nearer and nearer to the vertex of the bottom pebble, where the maximum heat transfer coefficient is found. As shown in Fig. 5d, it can be seen by comparing these 3 cases that a larger sphere brings about a stronger enhancement of local heat transfer coefficients at the left half of the diagonal plane; as for the right half of the plane, placing a sphere in strengthens the heat transfer around the contact points (case 1) compared to the beds without a sphere (cases 2 and 3). In addition, the significant effect of the sphere mainly focuses on the bottom pebble. That is quite reasonable because near there is located the spherepebble contact point.

\subsection{Velocity field in the gap}

The velocity field formed at the central gap of the middle plane is shown in Fig. 6a, b, and c, which help to understand the mechanism of heat transfer enhancement from another aspect. The white circles represent the inserted small spheres and by placing them in the void the flow field becomes more complex. The stagnant areas (blue area) near the front of the bottom pebble and the rear of the top pebble become smaller from case 1 to case 3 , therefore, increasing the diameter of the small sphere in the gap significantly reduces the stagnation area; in addition, the maximum flow velocity in the void increases from case 1 to case 3 , consequently, case 3 reasonably demonstrates the highest average HTC values. Furthermore, the velocity field on one side of the sphere is stronger than that on the other side (Fig. 6b and c), which affects the heat transfer near surrounding pebbles and causes different average HTCs as described in section 3.1. 

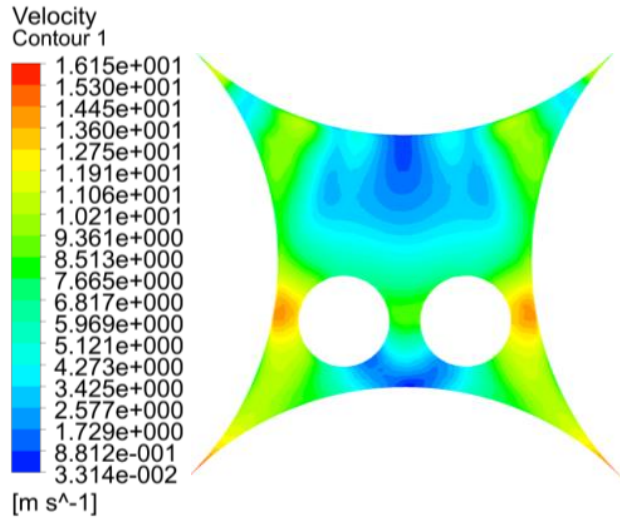

(a)

Velocity

$1.654 \mathrm{e}+001$

$1.567 \mathrm{e}+001$
$1.480 \mathrm{e}+001$

$1.480 \mathrm{e}+001$
$1.393 \mathrm{e}+001$

$1.306 \mathrm{e}+00$

$1.219 \mathrm{e}+001$

$1.132 \mathrm{e}+001$

$9.584 \mathrm{e}+000$

$9.584 \mathrm{e}+000$

$8.715 \mathrm{e}+000$

$7.846 \mathrm{e}+000$

$6.977 \mathrm{e}+000$

$5.238 \mathrm{e}+000$

$4.369 \mathrm{e}+000$

$3.500++000$

$2.631 \mathrm{e}+000$

$1.762 \mathrm{e}+000$
$8.930 \mathrm{e}-001$

$2.390 \mathrm{e}-002$

[m s^-1]

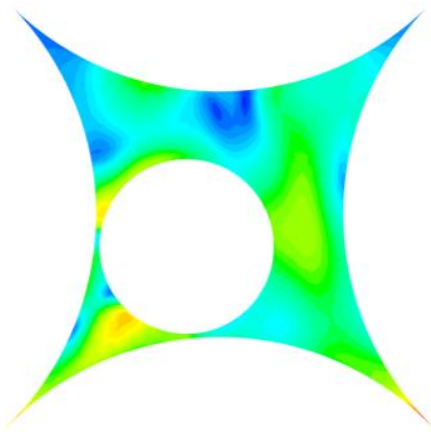

(b)

Velocity

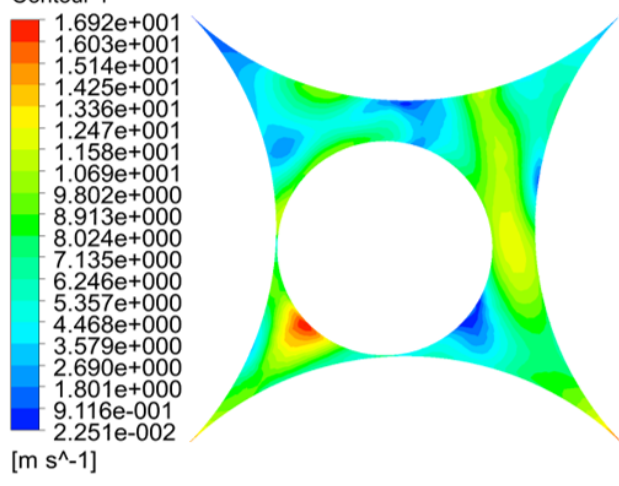

(c)

Fig. 6. Velocity field at the middle plane of the bed with (a) two spheres of $0.03 \mathrm{~m} \mathrm{(b)}$ a sphere of $0.0378 \mathrm{~m}$ and (c) a sphere of $0.0424 \mathrm{~m}$.

Fig. 7 shows the velocity field on the diagonal plane. The fast-flowing areas (red regions) and slowflowing areas were found respectively formed at the front and rear of pebble-pebble contact points. In addition, case 1 has the smallest maximum fluid velocity and the largest slow-flowing region (blue regions upper and lower two spheres in Fig. 7a), even though it contains two small spheres, while case 3 has the highest maximum velocity (Fig. 7c). Higher flow velocity means a better heat transfer, so the average HTC value of case 3 is the highest among the 3 cases. Furthermore, the smaller velocities in the area marked by the red square box explained the decrease of local HTC described in Fig. 5c and d.

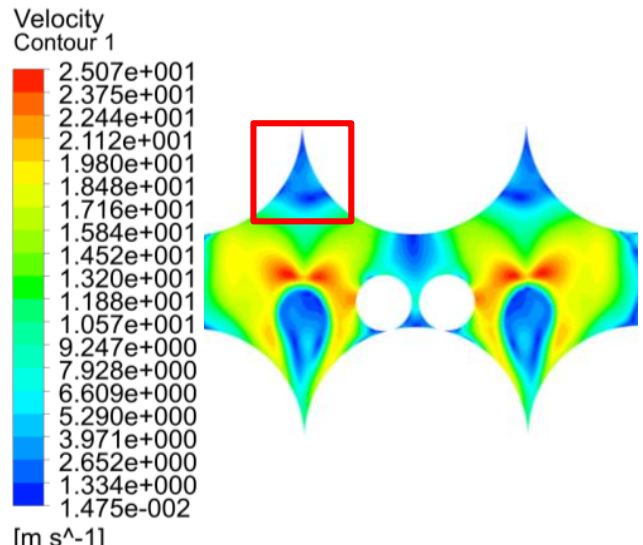

(a)

Velocity

Contour 1

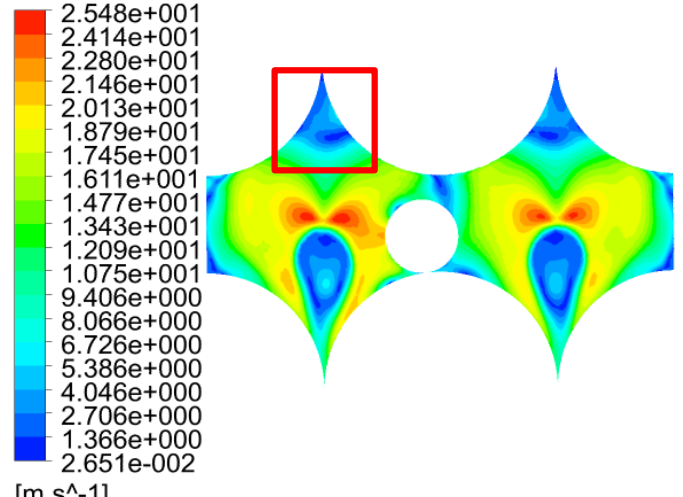

(b)

Velocity

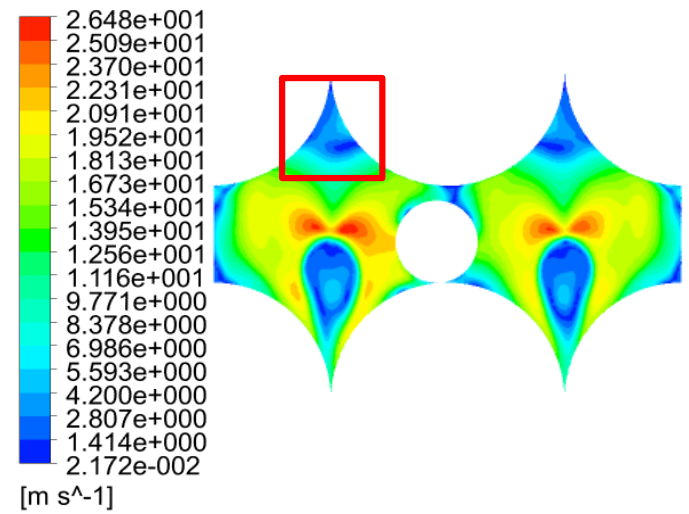

(c)

Fig. 7. Velocity field of the fluid at the diagonal planes for (a) two spheres of $0.03 \mathrm{~m}$ (b) a sphere of $0.0378 \mathrm{~m}$ and (c) a sphere of $0.0424 \mathrm{~m}$. 


\subsection{Surface temperature of pebbles}

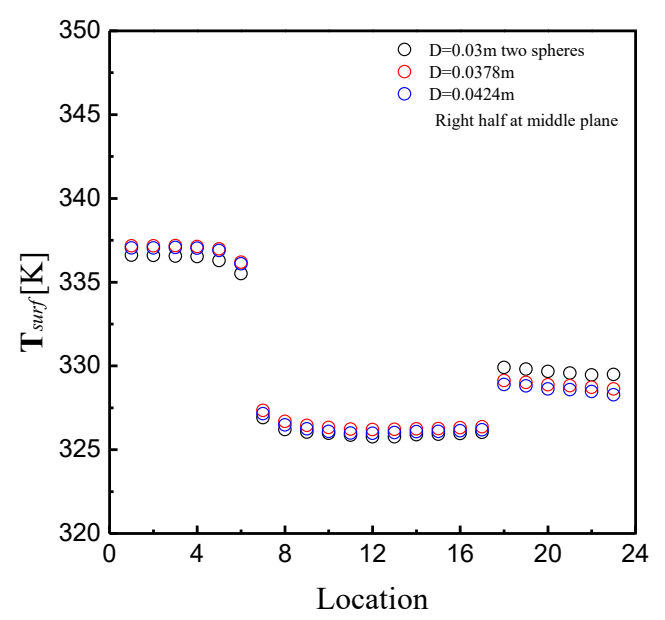

Fig. 8. Surface temperature of the designated pebbles

The surface temperatures of designated pebbles at the right half of the middle plane are shown in Fig. 8. The varying trend of all 3 cases is similar. Because a stagnant zone at the rear of the small spheres is formed in case 3 (Refer to Fig. 6a and Fig. 7a), the surface temperatures of the bottom pebble, therefore, are the highest among the 3 cases. One thing found in common for all 3 cases is that, compared to the pebble bed without small spheres, inserting spheres will reduce the surface temperature of adjacent pebbles, which indicates that inserting pebbles will reduce the possibility of hot spots.

The above discussion leads to a conclusion that placing more small spheres in a pebble bed does not necessarily improve the overall heat transfer performance compared to the pebble bed with only one inserted sphere that has the same volume or surface area. In reality, a larger sphere is recommended to be used to obtain better heat transfer characteristics. It is worth noting that although a larger sphere enhances the heat transfer around it more, in practical applications, the increasing pressure drop caused by the small sphere must be taken into account since it is also a key parameter of the core. According to the simulation results in the present study, the pressure drop between the inlet and outlet of the model for the bed with two spheres is $394 \mathrm{~Pa}$ and for the bed with a sphere diameter of $0.0424 \mathrm{~m}$ is $420 \mathrm{~Pa}$, the increasing ratio reaches $6.6 \%$. If the pressure drop is too large, it will demand a very high-power fan, there might be a possible safety hazard. Therefore, both the heat transfer characteristics and the fluid flow should be considered when designing a pebble bed core.

\section{Conclusions}

With the hope of obtaining the best structural parameters of a pebble bed, the effect of the quantity and size of the small spheres on the heat transfer characteristics of an FCC pebble bed was investigated in the present study. Particularly, two spheres with a diameter of $0.03 \mathrm{~m}$ were placed in the void of the pebble bed and their impact on the heat transfer characteristics was compared with the beds with only one sphere with a diameter of $0.0378 \mathrm{~m}$ or $0.0424 \mathrm{~m}$. The heat transfer coefficients on both the middle plane and the diagonal plane were measured in order to acquire more accurate results, and reasons for HTCs forming certain trends were analyzed as well. It is found that:

(1) When the volume of inserted spheres in different cases was kept the same, case $2(D=0.0378$ m) demonstrated a $1.4 \%$ higher HTC value than case 1 ; similarly, when the surface area of inserted spheres was kept constant, case $3(\mathrm{D}=0.0424 \mathrm{~m})$ showed a $2.8 \%$ increase of overall HTC value compared to case 1 ;

(2) The overall HTC showed an increasing trend with the ratio of the surface area to the volume decreasing, which differed from what the literature presented. The reason might be the inserted small spheres were fuel-free and only functioned as an expansion of the convective surface area;

(3) The maximum local HTCs were formed near pebble-sphere contact points, which validated the approach presented in this study.

Inserting a larger sphere into a pebble bed is preferred if only the heat transfer is considered when designing a pebble bed core, however, the pressure drop is also a key parameter and therefore deserves a deeper discussion in order to ensure the operational safety of a pebble bed reactor.

This work was supported by start-up young researchers fund (2019-BJ07) granted by Shaanxi University of Science \& Technology and was funded by the China Postdoctoral Science Foundation (No.2021M691999).

\section{References}

1. L. Chen, J. Lee, Ann. Nucl. Energy. 110, 338-348 (2017).

2. Z. Zhang, Y. Dong, F. Li, Z. Zhang, H. Wang, X. Huang, H Li, B. Liu, X. Wu, H. Wang, X. Diao, H. Zhang, J. Wang, Engineering 2, 112-118 (2016)

3. R. Moormann, R.S. Kemp, J. Li, Joule 2, 19111914 (2018)

4. L. Chen, J. Lee, Energy 212, 118642 (2020).

5. M.S. Latifi, G. Colangelo, G. Starace, Exp. Comput. Multiphas. Flow 2, 109-114 (2019)

6. C.W. Forsberg, P.F. Peterson, Nucl. Technol. 205, 748-754 (2019).

7. L. Chen, J. Lee, Appl. Therm. Eng. 172, 115151 (2020)

8. L. Chen, W. Lee, J. Lee, Appl. Therm. Eng. 121, 473-483 (2017)

9. L. Chen, J. Lee, E3S Web of Conferences, 2019, 128, 03004 (2019) 